\title{
EXPANDED TENSES IN THE OLD ENGLISH OROSIUS: A SYNTACTIC STRENGTHENING*
}

\subsection{EXPANDED TENSES}

2.1.1. The present chapter reports the investigation into certain aspects of the periphrastic construction to be + present participle (e.g. NE: "he is teaching "; OE. "he is laerende") viewed as an example of a syntactic strengthening. The construction is usually referred to as "continuous tenses/form" or "progressive tenses/form", whereas Nickel (1966) uses "expanded form". Coming closest to this latter term, the "expanded tenses" employed here seems a convenient label for two reasons:

a) the use of expanded tenses is not restricted to the expression of verbal aspect (Aspekt) or mode of verbal action (Aktionsart), which is implied by the use of either the term "continuous tenses/form" or the term "progressive tenses/form";

b) the expanded tenses are integrated into the English tense system, in the sense that they can be substituted with the respective non-expanded tenses without any change in the syntax of the clause, e.g.:

NE: "he is teaching in the temple" : "he teaches in the temple" OE: "he is laerende in temple" : "he laereb in temple"

In other words, we exclude constructions of the type "he is interesting" (with the participle having adjectival function) or "he is in the temple teaching" (with the participle acting as a weakened syntactic variant of the latter of the two implied clauses, "he is in the temple" and "he is teaching").

2.1.2. The assumption that Old English expanded tenses were felt as a syntactic strengthening can most easily be understood if they are compared with the corresponding non-expanded tenses:

a) the form of the expanded tenses is more elaborate than the form of the non-expanded tenses;

* The present paper summarizes the author's M.A. thesis "SYNTACTIC CHANGE. English expanded tenses". The M.A. thesis was written under the supervision of Professor Janez Orešnik during the author's M.A. studies at the Faculty of Arts in Ljubljana. It was defended at the same faculty on December 28, 1987. 
b) from the speaker's point of view, the expanded tenses are less economical than the non-expanded tenses;

c) from the hearer's point of view, the expanded tenses are easier to decode than the non-expanded tenses.

The expanded tenses are here viewed as due to the linguistic tendency to render communication clearer and more precise, which agrees with the definition of the term "strengthening" in Orešnik et al. (in the present volume). The following predictions can be derived from the assumptions stated above:

a) that the development of expanded tenses in Old English followed the course outlined for "strengthenings" in Orešnik et al. (in the present volume);

b) that on the synchronic level expanded tenses act as a strengthened construction vis-a-vis non-expanded tenses, in terms of form, meaning and frequency.

2.1.3. The construction composed of to be + present participle, as well as the combinations of other copulas with participles, gerunds and agent nouns, were known to Hittite, Avestan and Old Indian, to Old Greek and Latin, and they are commonly used in modern Spanish, Italian and English. Although the actual usage differs from language to language, such constructions are not alien to any Indo-European language (Mossé 1938, I, 1-18; Nickel 1966, 59-82). ${ }^{1}$

2.1.4. Expanded tenses (the periphrastic construction beon/wesan + present participle ending in -ende) can be found in the oldest extant Old English manuscripts. While infrequent in the so-called "pagan poetry" (only four instances in Beowulf, ed. Klaeber 1950), they are relatively frequent in texts translated from Latin. Mossé went as far as to ascribe to the influence of Latin, in which such constructions were quite common, the introduction of expanded tenses into the Old English language:

"Quelles que soient leur nature, leur dates et leur origine, poésie ou prose, traductions ou originaux, tous les textes du vieil anglais nous ont donnés des clercs, c'est-à-dire des lettrés qui tous savaient le latin. "(Mossé 1938, I, 53-54).

Nickel dismissed this interpretation $(1966,83-207)$. He compared the Old English translations with the respective Latin originals and found no correlation between the use of expanded tenses in Old English and of corresponding constructions in the originals, although word-to-word translations often obscure the real frequency of expanded tenses in Old English.

In some languages this construction exists merely as a potential option (Slovene "bil sem pojoč"), in other languages it occurs as a strengthened variant of usually imperfective verbal constructions (Spanish "Juan estaba llegando" vs. "Juan llegaba") or else it conveys the meaning of a specific grammatical category within the verbal paradigm. In this last instance, its usage is regularized and does not display the behaviour of strengthened syntactic variants. 
Mossé saw another reason for the use of expanded tenses in Old English in the disappearance of the old Germanic system of verbal aspects. ${ }^{2}$ Due to the implicit durative value of the present participle, expanded tenses, according to Mossé, were used to express the imperfective verbal aspect. Mossé, however, failed to explain the surprisingly high incidence of expanded tenses in identical contexts and situations to those where non-expanded tenses are used. That incidence made Nickel disagree with Mossé. The present author, however, believes that Mossé's point of view is valid for the following reasons:

a) geographically, expanded tenses spread in the direction in which the former system of verbal aspects disappeared, i.e. from the north towards the south of England (Mossé 1938, II, 1-38);

b) the use of expanded tenses in Old English gained grounds especially after the year 1200 , when the prefix ge-, formerly marking perfectiveness, disappeared even from the southern dialects (Mossé ibid.);

c) according to CONSTRUCTIONAL ICONISM (Mayerthaler 1980,; a language tends to express marked categories with markful forms. From this point of view, the prefixation of verbs with ge- to express some mode of action (Aktionsart) would be "normal" (stable). Once the prefix had lost its lexical meaning and began to denote perfective verbal aspect (Aspect) instead of the mode of action (Aktionsart), the situation was reversed: a markful form was used to express an unmarked category. This "anomaly" led to the weakening of the prefix until verb pairs such as" "feohtan" and "gefeohtan" became interchangeable. The ensuing systemic instability (absence of aspectual distinction) led to a further syntactic change (i.e. to the assertion of expanded tenses in the verbal paradigm). This speculation deserves further exploration.

2.1.5. The origin of English expanded tenses is irrelevant from the standpoint of our working hypothesis. It hardly matters which one of the numerous factors (the influence of Latin, the disappearance of the former system of verbal aspect, the analogy with constructions such as beon/wesan + agent nouns -end or gerund) played the main role. On the basis of the theory propounded in Orešnik et al. (in the present volume) we asume,

a) that at a given period of time and under given circumstances the existing verbal paradigm was felt as insufficient and a new "stronger" element of the paradigm was called for and eventually added (i.e. expanded tenses);

2 In the present report verbal aspect (Aspekt) refers solely to the dichotomy of verbs viewing a situation from the standpoint of its internal structure (imperfective aspect). The mode of verbal action (Aktionsart) refers to the stage (phase) of action which is brought into focus (ingressive, perfective, resultative, progressive etc.). 
b) that expanded tenses initially asserted themselves under relatively complex grammatical conditions, in a complex environment.

Eventually, the expanded tenses began to express "marked information" only, whereby their usage became regularized. This part of the development was due to the dissimilation of the expanded tenses vis-a-vis the non-expanded tenses.

\subsection{THE ANALYSIS OF THE OROSIUS}

2.2.1. The working hypothesis stated in Orešnik et al. (in the present volume) has been tested on the Old English expanded tenses in King Alfred's Old English translation of the Latin work "Historiarum Adversum Paganos".

The author of the original, a Spanish monastic Orosius, was induced by the African bishop St. Augustine to write a history of the World, from the creation to A.D. 416, upon Christian principles, to show "the real origin of the misery of the World" (Bosworth 1859, iv).

According to Asser (Wise 1772), Alfred began to translate from Latin into Old English in 887 , Orosius being his third translation finished probaly about the year 890. The translation is rather free. Not only did Afred omit what he deemed of little importance, he also added his own remarks and observations and inserted original instructive essays (the descriptions of Europe, the voyages of Ohthere and Wulfstan, etc.).

The choice of this work for analysis was dictated by the independence of the translation and by the relatively high frequency of expanded tenses in it (Mossé 1938, I, 75-76). The syntactic independence of the translation seemed of particular importance; of 237 instances of expanded tenses in the Old English version, 53 correspond to non-expanded (simple) tenses in the original text (Mossé 1938, 66), and as many as 78 have no proper equivalent in the Latin original (Nickel 1966, 112).

2.2.2. Our -investigation of the Orosius involves the comparison of two samples, the basic sample and the control sample. The basic sample consists of 237 clauses containing instances of expanded tenses in the edition (Sweet 1883, repr. 1956). The control sample consists of 855 randomly chosen clauses containing instances of non-expanded tenses in the edition (every third clause on every third page of the edition has been analyzed). The relative density of the control sample was dictated by the stylistic and syntactic unevenness of the text.

The clauses thus identified have been analyzed in terms of their type and propositional modality; the verbal phrases contained in those clauses have been analyzed as to tense, mood, number and transitivity. The choice of parameters just mentioned 
was dictated by the properties of the basic sample (e.g. person and voice are not included since the basic sample does not contain any instances of non-third persons or of non-active voice). The analysis of the clauses provides some (partial) information about how complex the conditions are under which (non-)expanded tenses occur in Orosius. To calculate the probability that a given parameter assumes its marked value in the expanded and non-expanded tenses, the following formula has been used:

$$
\mathrm{P}_{(\mathrm{a})}=\mathrm{n}_{(\mathrm{a})} / \mathrm{n}^{3}
$$

For the working hypothesis to be confirmed, the probability rates of favourable events must be higher in the basic sample than in the control sample:

$$
P_{(a)}>P_{(b)}
$$

The index which shows whether the difference bettween any two compared probability rates is statistically significant, has been calculated with the aid of the formula:

$$
\text { Id }=\frac{P_{(a)}-P_{(b)}}{s d}
$$

sd (standard deviation) being,

$$
s d=\sqrt{\frac{\hat{\mathrm{P}} \times \overrightarrow{\mathrm{q}\left(\mathrm{n}_{(\mathrm{a})}+\mathrm{n}_{(\mathrm{b}))^{5}}\right.}}{\mathrm{n}_{(\mathrm{a})} \times \mathrm{n}_{(\mathrm{b})}}}
$$

Two probability rates are statistically significantly different if the value of the index Id exceeds 2 (Pavlić 1985).

In a given clause, some parameters may assume a marked value and others not. We predict that the probability for more parameters to assume a marked value will be higher in the sample of expanded tenses than in the sample of non-expanded tenses. This "cumulative markedness" has been calculated with the help of the median and quartiles. ${ }^{6}$

$3 \mathrm{a}=$ favourabe event in the basic sample, in our case the presence of the more complex (marked) form of a parameter (e.g. preterite instead of present);

$P_{(a)}=$ probability of favourable event

$\mathrm{n}_{(\mathrm{a})}=$ total number of favourable events

$\mathrm{n}=$ total number of all possible events

$4 b_{(a)}=$ favourable event in the control sample (i.e. the presence of the marked form a parameter)

$5 \quad \overline{\mathbf{P}}^{\prime}=$ mean probability rate;

$\bar{q}=1-\overline{\mathrm{p}}$

6. median $=$ the value below and above which $50 \%$ of the observed values are distributed; quartile $=$ the first (third) quartile is the value under which $25 \%(75 \%)$ of all the observed values are distributed. 
Furthermore, we have calculated the arithmetical mean of the probability rates in both samples. For our hypothesis to be confirmed, the arithmetical mean of the probability rates of favourable events must be higher in the basic sample than in the control sample.

\subsection{THE STATISTICAL ANALYSIS AND RESULTS ${ }^{7}$}

\subsubsection{The analysis of the clauses contained in the basic sample ${ }^{8}$}

\section{$2 / 25$ waeron winnende}

C: $\mathrm{m}+$; T: pret.; M: ind.; A: imp.; Tr: prep.; N: pl.

NE.: had wars, warred

\section{3/32 waes giernende}

C: $\mathrm{m}+$; T: pret.; M: ind.; A: imp.; Tr: gen.; N: sing.

NE.: affected, was desirous

\section{6/7 waes sprecende}

C: $\mathrm{m}+$; T: pret.; M: ind.; A: imp.; Tr: prep.; N: sing.

NE.: said, was speaking

\section{$8 / 15$ is irnende}

C: ambiguous +; T: pres.; M: ind.; A: imp.; Tr: intran.; N: sing. Ne.: takes its source, takes its source

\section{$12 / 22$ sie irnende}

C: sub.+ ; pres.; M: subj.; A: imp.; Tr: intran.; N: sing.

NE.: flows, flows running

7 This section contains the grammatical analysis of the clauses contained in the basic sample (a similar analysis of the clauses contained in the control sample is not repeated here for reasons of economy) followed by the results of the statistical work.

8 For brevity sake, not whole clauses, but only their expanded tenses are quoted; the figure introducing each example indicates the page and the line (of the edition) in which the instance of the expanded tense occurs.

$\mathrm{C}=$ type of clause and propositional modality; $\mathrm{T}=$ tense; $\mathrm{M}=\operatorname{mood} ; \mathrm{A}=$ aspect; $\mathrm{Tr}=$ transitivity; $\mathrm{N}=$ number;

$\mathrm{m}=$ main clause, sub. $=$ subordinate clause $++=$ affirmative propositional modality, $-=$ negative propositional modality, ? = interrogative propositional modality; pret. = preterite; pres. = present; subj. = subjunctive; ind. = indicative mood; imp. = imperfective aspect; perf. = perfective aspect; intran. = intransitive, gen. = with genitive, dat. = with dative; acc. = with accusative; prep = with prepositional object; obj.c. $=$ with object clause; pl. = plural; sg. = singular;

NE. = the Modern English translation of the expanded tense taken from Barrington's (1773) and Thorpe's (1873) translations of Orosius into Modern English. Both translations are quoted in Nickel $(1966,330-351)$. In matters of aspect, Nickel's judgement has been followed (1966, ibid). 
$12 / 23$ sie flowende

C: sub.+; T: pres.; M: subj.; A: imp.; Tr: intran.; N: sing. NE.: proceeds, flows up

$12 / 26$ is irnende

C: $\mathrm{m}+$; T: pres.; M: ind.; A.: imp.; Tr: intran.; N: sing.

NE.: runs, runs

\section{2/35 bid flowende}

C: sub. +; T: pres.; M: ind.; A: imp.; Tr: intran.; N: sing. NE.: is spread, is flowing

\section{$14 / 32$ is irnende}

C: $\mathrm{m}+$; T: pres.; M: ind.; A: imp.; Tr: intran.; N: sing.

NE.: runs, runs

\section{9/34 waes yrnende}

C: sub. +; T: pret.; M: ind.; A: imp.; Tr: intran.; N: sing.

NE.: being under sail, was running

\section{8/28 waes heriende7feohtende}

$\mathrm{C}: \mathrm{m}+$; T:ß pret.; M: ind.; A: imp.; Tr: intran.; N: sing.

NE.: committed devastations, committed devastations

C: $\mathrm{m}+$; T: pret.; M: ind.; A: imp.; Tr: intran.; N: sing.

NE.: continued to carry on wars, carried on war

30/5 onwinnende waes

C: sub. +; T: pret.; M: ind.; A: imp.; Tr: dat.; N: sing.

NE.: by...making war, by...making war

$30 / 12$ waes feohtende

C: $\mathrm{m}+$; T: pret.; M: ind.; A: imp.; Tr: prep.; N: sing.

NE.: was attacking, was fighting

30/17 waes dreogende

C: $\mathrm{m}+$; T: pret.; M: ind.; A: imp.; Tr: acc.; N: sing.

NE.: continued, carried on

30/19 waes feohtende

C: $\mathrm{m}+$; T: pret.; M: ind.; A: imp.; Tr: prep.; N: sing.

NE.: attacked, made war on 
$30 / 21$ waes wilniende

C: $\mathrm{m}$ + ; T: pret.; M: ind.; A: imp.; Tr: obj.c.; N: sịng.

NE.: was desirous, was desirous

$30 / 27$ fremmende waes

C: $\mathrm{m}+$; T: pret.; M: ind.; A: imp.; Tr: acc.; N: sing.

NE.: wished to prostitute, was...perpertrating

32/38 waes brucende

$\mathrm{C}: \mathrm{m}+$; T: pret.; M: ind.; A: imp.; Tr: gen.; N: sing.

NE.: were enjoying, was enjoying

$32 / 11$ waes standende

C: $\mathrm{m}+$; T: pret.; M: ind.; A: imp.; Tr: intran.; N: sing.

NE.: a flood covered, was standing

32/29 waeran singende

C: $\mathrm{m}+$; T: pret.; M: ind.; A: imp.; Tr: prep.; N: pl.

NE.: give this account, thus sang

34/16 waes secgende

C: $\mathrm{m}+$; T: pret.; M: ind.; A: imp.; Tr.: obj.c.; N: sing.

NE.: say, says

36/31 binnende waeron

C: ambiguous +; T: pret.; M: ind.; A: imp.; Tr: acc.; N: pl.

NE.: pained, were pained

38/7 waeron berstende 7 (...) utsionde

C: $\mathrm{m}+$; T: pret.; M: ind.; A: imp.; Tr: intran.; N: pl.

NE.: burned, burst

C: $\mathrm{m}+$; T: pret.; M: ind.; A: imp.; Tr: acc.; N: pl.

NE.: issued, issued

$38 / 12$ sceorfende waeron

C: sub. +; T: pret.; M: ind.; A: imp.; Tr: acc.; N: pl.

NE.: destroyed, gnawed off

$38 / 22$ waes aefterfylgende

C: $\mathrm{m}+$; T: pret.; M: ind.; A: imp.; Tr: dat.; N: sing.

NE.: determined to pursue, was...following 
38/35 gongende waeron

C: sub.+; T: pret.; M: ind.; A: imp.; Tr: intra.; N: pl. NE.: passed, passed

40/26 donde waeron

C: ambiguous + ; T: pret.; M: ind.; A: imp.; Tr: acc.; N: pl.

NE.: guilty of...murders, were enacting...such murders

40/32 winnende waes

C: $\mathrm{m}+$; T: pret.; M: ind.; A: imp.; Tr: prep.; N: sing.

NE.: made wars, made war

42/6 donde waeron

C: sub.+; T: pret.; M: ind.; A: imp.; Tr: acc.; N: pl.

NE.: which befell them, that they did

42/31 waeron winnende

C: $\mathrm{m}+$; T: pret.; M: ind.; A: imp.; Tr: prep.; N: pl.

NE.: wars, warred

42/33 beon feohtende

C: infinitive, A: imp.; Tr: prep.

NE.: fighting, fighting

44/4 waes winnende

C: $\mathrm{m}+$; T: pret.; M: ind.; A: imp.; Tr: intran.; N: sing.

NE.: carried on a war, carried on a war

44/6 waes farende

C: $\mathrm{m}+$; T: pret.; M: ind.; A: imp.; Tr: intran.; N: sing.

NE.: marched, marched

44/16 aefterfylgende waeron

C: $\mathrm{m}+$; T: pret.; M: ind.; A: imp.; Tr: dat.; N: pl.

NE.: pursued, pursued

44/19 waeron heriende 7 westende

C: $\mathrm{m}+$; T: pret.; M: ind.; A: imp.; Tr: acc.; $\mathrm{N}: \mathrm{pl}$.

NE.: continued to...plunder, were plundering

C: $\mathrm{m}+$; T: pret.; M: ind.; A: imp.; Tr: acc.; N: pl.

NE.: continued to...plunder, were ravaging 
44/27 winnende waeron

C: $\mathrm{m}+$; T: pret.; M: ind.; A: imp.; Tr: intran.; N: pl.

NE.: continued, carried on war

46/5 winnende waeron 7 (...) sleande

C: $\mathrm{m}+$; T: pret.; M: ind.; A: imp.; Tr: prep.; N: pl.

NE.: carrying on the war, carried on the war

C: $\mathrm{m}+$; T: pret.; M: ind.; A: imp.; Tr: acc.; N: pl.

NE.: put them to death, slew

48/10 wendende waeron

C: $\mathrm{m}+$; T: pret.; M: ind.; A: imp.; Tr: acc.; $\mathrm{N}$ : pl.

NE.: governed, turned

48/23 biddende sindon

C: sub. +; T: pres.; M: ind.; A: imp.; Tr: prep./gen.; N: pl.

NE.: was now obliged, was...begging

$50 / 3$ donde waeron

C: sub.+; T: pret.; M: ind.; A: imp.; Tr: acc.; N: pl.

NE.: spread such devastations, when...did...evil

$50 / 13$ sittende waeron 7 feohtende

C: $\mathrm{m}+$; T: pret.; M: ind.; A: imp.; Tr: intran.; N: pl.

NE.: continued the siege, were...investing

C: $\mathrm{m}+$; T: pret.; M: ind.; A: imp.; Tr: intran.; N: pl.

NE.: continued, were fighting

50/18 dreogende waeron

C: sub.+; T: pret.; M: ind.; A: imp.; Tr: acc.; N: pl.

NE.: happened, -

$50 / 23$ waes aefterfylgende

C: $\mathrm{m}+$; T: pret.; M: ind.; A: imp.; Tr: intran.; N: sing.

NE.: ensued, ensued

54/1 iernende /waeron/

C: $\mathrm{m}+$; T: pret.; M: ind.; A: imp.; Tr: intran.; N: pl.

NE.: hasted, running

54/9 waes forsacende

C: $\mathrm{m}+$; T: pret.; M: ind.; A: perf.; Tr: acc.; N: sing.

NE.: renounced, destroyed 
$54 / 18$ waes cwielmende

C: $\mathrm{m}+$; T: pret.; M: ind.; A: imp.; Tr: acc.; N: sing.

NE.: oppressed, destroyed

54/22 donde waes

C: sub. +; T: pret.; M: ind.; A: imp.; Tr: acc.; N: sing.

NE.: in tormenting, he was inflicting

54/26 prowiende waeron

C: $\mathrm{m}+$; T: pret.; $\mathrm{M}$ : ind.; A: imp.; Tr: acc.; N: pl.

NE.: - , were suffering

56/1 waere donde

C: sub. +; T: pret.; M: subj.; A: imp.; Tr: acc.; N: sing.

NE.: were...doing evils, -

$56 / 8$ winnende waeron

C: $\mathrm{m}+$; T: pret.; M: ind.; A: imp.; Tr: prep.; N: pl.

NE.: happened the wars, carried on war

56/11 waeron winnende

C: $\mathrm{m}+$; T: pret.; $\mathrm{M}$ : ind.; A: imp.; Tr: intran.; N: pl.

NE.: made incursions, made war

$56 / 15$ winnende waeron

C: $\mathrm{m}+$; T: pret.; M: ind.; A: imp.; Tr.: prep.; N: pl.

NE.: were at war, were at war

$56 / 26$ sittende waeron

C: $\mathrm{m}+$; T: pret.; M: ind.; A: imp.; Tr: intran.; N: pl.

NE.: whilst...beseiged, should beseige

58/4 dreogende waeron

C: $\mathrm{m}+$; T: pret.; M: ind.; A: imp.; Tr: intran.; N: pl.

NE.: continued at war, suffered

58/17 wrecende waes

C: $\mathrm{m}+$; T: pret.; M: ind.; A: imp.; Tr: acc.; N: sing.

NE.: shewed his wrath, avenged it

58/30 ricsiende sindon

C: sub.+; T: pres.; M: ind.; A: imp.; Tr: intran.; N: pl.

NE.: continues, are still ruling 
$62 / 30$ ricsiende sindon

C: $\mathrm{m}+$; T: pres.; M: ind.; A: imp.; Tr: intran.; N: pl. NE.: continue, are now yet ruling

$64 / 2$ settende is 7 wendende

C: sub.+; T: pres.; M: ind.; A: imp.; Tr: acc.; N: sing.

NE.: continues to do, yet establishes

C: sub.+; T: pres.; M: ind.; A: imp.; Tr: acc.; N: sing.

NE.: continues, turns

64/8 libbende waeran

C: $\mathrm{m}+$; $\mathrm{T}$ : pret.; M: ind.; A: imp.; Tr: intran.; N: pl. NE.: persisted, were living

64/11 bowiende waeron

C: $\mathrm{m}+$; T: pret.; M: ind.; A: imp.; Tr: dat.; N: pl.

NE.: served, served

64/24 stellende waes

C: $\mathrm{m}+$; T: pret.; M: ind.; A: imp.; Tr: acc.; N: sing.

NE.: shewed, set

66/1 iernende waeron

C; sub.+; T: pret.; M: ind.; A: imp.; Tr: intran.; N: pl.

NE.: ran, ran

66/2 waeron feallende 7 biddende

C: sub. +; T: pret.; M: ind.; A: imp.; Tr: intran.; N: pl.

NE.: falling, fell

C: sub. +; T: pret.; M: ind.; A: imp.; Tr: obj.c.; N: pl. NE:: intreated, intreated

66/18 boliende waeron

C: sub. +; T: pret.; M: ind.; A: imp.; Tr: acc.; N: pl.

NE.: suffered, were suffering

$66 / 21$ winnende waeron

C: $\mathrm{m}+$; T: pret.; M: ind.; A: imp.; Tr: intran.; N: pl.

NE.: continued their attacks, continued the war

$66 / 23$ winnende waeron

C: $\mathrm{m}+$; T: pret.; $\mathrm{M}$ : ind.; A: imp.; Tr: prep.; N: pl.

NE.: warred, warred 
68/31 dreogende waes

C: sub. +; T: pret.; M: ind.; A: imp.; Tr: acc.; N: sing.

NE.: after he had been carrying, which he had been carrying

70/1 ondraedende waeron

C: $\mathrm{m}+$; T: preet.; M: ind.; A: imp.; Tr: acc.; N: pl.

NE.: alarmed, dreaded

$70 / 11$ brociende waeron

C: $\mathrm{m}+$; T: pret.; M: ind.; A: imp.; Tr: acc.; N: pl.

NE.: continued to afflict, continued to afflict

$72 / 2$ waeron wuniende

C: $\mathrm{m}+$; T: pret.; M: ind.; A: imp.; Tr: intran.; N: pl.

NE.: was...resident, dwelt

$74 / 3$ is irnende

C: sub. +; T: pres.; M: ind.; A: imp.; Tr: intran.; N: sing.

NE.: runs, runs

74/6 farende waes

C: $\mathrm{m}+$; $\mathrm{T}$ : pret.; $\mathrm{M}$ : ind.; A: imp.; Tr: intran.; $\mathrm{N}$ : sing.

NE.: passing, proceeded

$74 / 18$ is irnende

C: sub. +; T: pres.; M: ind.; A: imp.; Tr: intran.; N: sing.

NE.: is running, runs

$74 / 25$ sprecende sie

C: sub. +; T: pres.; M. subj.; A: imp.; Tr: prep.; N: sing.

NE.: addresses, as if it were speaking

74/33 waes aefterfylgende

C: $\mathrm{m}+$; T: pret.; $\mathrm{M}$ : ind.; A: imp.; Tr: dat.; N: sing.

NE.: whilst...were behind, while...was following

$76 / 2$ is hreosende

C: sub. +; T pres.; M: ind.; A: imp.; Tr: intran.; N: sing.

NE.: are...decaying, are falling

$76 / 5$ wenende waes

C: $\mathrm{m}+$; T: pret.; M: ind.; A: imp.; Tr: ob.c.; N: sing.

NE.: -, imagined 
$76 / 5$ fleonde waeron

C: sub. +; T: pret.; M: ind.; A: imp.; Tr: intran.; N: pl.

NE.: - , were fleing

76/18 drincende waeron

C: $\mathrm{m}+$; $\mathrm{T}$ : pret.; $\mathrm{M}$ : ind.; A: imp.; Tr: acc.; N: pl.

NE.: drank, drank

$76 / 20$ waes farende

C: $\mathrm{m}+$; T: pret.; M: ind.; A: imp.; Tr: intran.; $N$ : sing.

NE.: marched, went

$76 / 21$ wunniende waes

C: sub. +; T: pret.; M: ind.; A: imp.; Tr: intran.; N: sing.

NE.: was dwelling, was staying

76/24 Dencende waes

C: $\mathrm{m}+$; T: pret.; M: ind.; A: imp.; Tr: prep./obj.c.; N: sing.

NE.: bethought herself, was...thinking

76/28 farende waes

C: sub.+; T: pret.; M: ind.; A: imp.; Tr: intran.; N: sing.

NE.: was flying, were fleeing

$76 / 30$ waes aefterfylgende

C: $\mathrm{m}+$; T: pret.; M: ind.; A: imp.; Tr: dat.; N: sing.

NE.: whilst...were behind, while...was following

76/33 pyrstende waere

C: sub.+; T: pret.; M: subj.; A: imp.; Tr: ten.; N: sing.

NE.: hast been thirsting, hast been thirsting

$80 / 25$ feohtende waeron

$\mathrm{C}: \mathrm{m}+$; T: pret.; M: ind.; A: imp.; Tr: intran.; N: pl.

NE.: groaning, was...groaning

80/34 waes sprecende 7 geormiende

C: $\mathrm{m}+$; T: pret.; $M$ : ind.; A: imp.; Tr: intran.; N: sing.

NE.: addressed, was speaking

C: $\mathrm{m}+$; T: pret.; M: ind.; A: imp.; Tr: intran.; N: sing.

NE.: groaning, was groaning 
82/7 waes wilniende

C: $\mathrm{m}+$; T: pret.; M: ind.; A: imp.; Tr: obj.c.; N: sing. NE.: flattered himself, was...desirous

$82 / 12$ feohtende waeron

C: $\mathrm{m}+$; T: pret.; $\mathrm{M}$ : ind.; A: imp.; Tr: intran.; N: pl.

NE.: - , -

82/21 bidde(nde) waes

C: $\mathrm{m}+$; T: pret.; $\mathrm{M}$ : ind.; A: imp.; Tr: obj.c.; $\mathrm{N}$ : sing. NE.: prevailed, besought

$82 / 26$ fleonde waeron

C: $\mathrm{m}+$; T: pret.; M: ind.; A: imp.; Tr: intran.; N: pl.

NE.: fled, took to flight

$82 / 29$ waes laerende

C: $\mathrm{m}+$; T: pret.; $\mathrm{M}$ : ind.; A: imp.; Tr: obj.c.; $\mathrm{N}$ : sing.

NE.: advised, advised

$84 / 9$ waes ondraedende

C: $\mathrm{m}+$; T: pret.; M: ind.; A: imp.; Tr: obj.c.; N: sing.

NE.: - , -

84/9 waeren aefterfylgende

C: sub. +; T: pret.; M: subj.; A: imp.; Tr: dat.; N: pl.

NE.: being in pursuit, were in pursuit

84/14 waes biddende

C: sub. +; T: pret.; M: ind.; A: imp.; Tr: gen.; N: sing.

NE.: was now obliged, was...begging

84/17 farende waeron

C: sub.+; T: pret.; M: ind.; A: imp.; Tr: intran.; N: pl.

NE.: the fleet, in which they had been faring

$84 / 33$ sprecend(e) waes

C: sub +; T: pret.; M: ind.; A: imp.; Tr: prep.; N: sing.

NE.: spoke, said

$86 / 23$ birnende waere

C: sub. +; T: pret.; M: subj.; A: imp.; Tr: intran.; N: sing.

NE.: as if on fire were burning 
$86 / 30$ winnende waeron

C: $\mathrm{m}+$; pret.; M: ind.; A: imp.; Tr: prep.; N: pl.

NE.: made war, made war

88/11 waes cwaciende 7 berstende

C: $\mathrm{m}+$; T: pret.; M: ind.; A: imp.; Tr: intran.; N: sing.

NE.: continued to quake, quaked

C: $\mathrm{m}+$; T: pret.; $\mathrm{M}$ : ind.; A: imp.; Tr: intran.; N: sing.

NE.: continued to burst, burst

$88 / 20$ waes on teonde

C: ambiguous + ; T: pret.; M: ind.; A: imp.; Tr: dat./acc.; N: sing.

NE.: arrogated, would arrogate

88/21 gehafiende naeren

C: sub.—; T: pret.; M: subj.; A: imp.; Tr: gen./dat.; N: pl.

NE.: allowed, would not concende

$88 / 22$ winnende waeron

C: $\mathrm{m}+$; T: pret.; M: ind.; A: imp.; Tr: prep.; N: pl.

NE.: - , -

$88 / 26$ wergende waeron

C: $\mathrm{m}+$; T: pret.; M: ind.; A: imp.; Tr: acc.; N: pl.

NE.: banished, defended

90/8 winnende waeron

C: sub. +; T: pret.; M: ind.; A: imp.; Tr: prep.; N: pl.

NE.: had perpetual wars, had...warred

90/10 winnende waeron

C: $\mathrm{m}+$; T: pret.; M: ind.; A: imp.; Tr: prep.; N: pl.

NE.: were at war, made war

90/18 dreogende waeron

C: $\mathrm{m}+$; T: pret.; M: ind.; A: imp.; Tr: acc.; N: pl.

NE.: continued, carried on

92/14 waeron aefterfylgende

C: $\mathrm{m}+$; T: pret.; M: ind.; A: imp.; Tr: dat.; $\mathrm{N}$ : pl.

NE.: pursued, followed 
92/15 waeron hergende

C: $\mathrm{m}+$; T: pret.; M: ind.; A: imp.; Tr: acc.; N: pl.

NE.: devastation, ravaged

92/16 waeron sleande

C: $\mathrm{m}+$; T: pret.; M: ind.; A: imp.; Tr: ac.; N: pl.

NE.: it was filled with every kind of devastation, slaughter

94/1 naeron hergende

C: $\mathrm{m}-$; T: pret.; M: ind.; A: imp.; Tr: prep.; N: pl.

NE.: plundered, were plundering

92/2 waeron hergende 7 (...) baernende

C: $\mathrm{m}+$; T: pret.; M: ind.; A: imp.; Tr: intran.; N: pl.

NE.: ransacked the city, were...ravaging

C: $\mathrm{m}+$; T: pret.; M: ind.; A: imp.; Tr: acc.; N: pl.

NE.: ransacked, were burning

94/9 waeron wilniende

C: $\mathrm{m}+$; T: pret.; M: ind.; A: imp.; Tr: obj.c.; N: pl.

NE.: chose, were...desirous

94/29 giddende sindon

C: sub.+; T: pres.; M: ind.; A: imp.; Tr: intran.; N: pl. NE.: panegyrics, sing

98/16 waeron ondraedende

C: $\mathrm{m}+$; T: pret.; M: ind.; A: imp.; Tr: obj.c.; N: pl.

NE.: began to dread, dreaded

$100 / 1$ waeron winnende

C: $\mathrm{m}+$; T: pret.; M: ind.; A: imp.; Tr: prep.; N: pl.

NE.: made war, made war

$100 / 29$ waeron winnende

C: sub.+; T: pret.; M: ind.; A: imp.; Tr: prep.; N: pl.

NE.: had been at war, had been warring

$100 / 31$ waeron hergende

C: $\mathrm{m}+$; T: pret.; M: ind.; A: imp.; Tr: prep.; N: pl.

NE.: laid waste, laid waste 
104/15 winnende waeron

C: sub.+; T: pret.; M: ind.; A: imp.; Tr: prep.; N: pl.

NE.: - , they were warring

104/18 weaxende waes

C: ambiguous + ; T: pret.; M: ind.; A: imp.; Tr: intran.; N: sing.

NE.: ensued, went on increasing

104/19 dypende waeron

C: sub.+; T: pret.; M: ind.; A: imp.; Tr: obj.c.; N: pl.

NE.: appeared, testified

106/13 winnende beon

C: infinitive, A: imp.; Tr: intran.

NE.: carried on their wars, would be at war

108/29 donde waeron

C: $\mathrm{m}+$; T: pret.; M: ind.; A: imp.; Tr: acc.; N: pl.

NE.: continued this, this they did

$110 / 11$ gemunende beon

C: infinitive; A: imp.; Tr: gen.

NE.: will take notice, will...mention

$112 / 21$ waeron winnende

C: $\mathrm{m}+$; T: pret.; $\mathrm{M}$ : ind.; A: imp.; Tr: prep.; $\mathrm{N}$ : pl.

NE.: -, were...warring

$112 / 24$ winnende waes

C: sub. +; pret.; M: ind.; A: imp.; Tr: prep.; N: sing.

NE.: enemies, were warring

$112 / 29$ beowiende waeron

C: sub +; T: pret.; M: ind.; A: imp.; Tr: dat.; N: pl.

NE.: were under his power, were his slaves

\section{8/1 waes cirrende}

C: sub.+; T: pret.; M: ind.; A: imp.; Tr: intran.; N: sing.

NE.: intended to return, was on his return

118/19 waes hergende

C: $\mathrm{m}+$; T: pret.; M: ind.; A: imp.; Tr: prep.; N: sing.

NE.: advanced, made hostila inroads 
118/20 waes sierwende

C: $\mathrm{m}+$; T: pret.; M: ind.; A: imp.; Tr: intran.; N: sing.

NE.: - , laid ambushes

120/12 waes hienende

C: $\mathrm{m}+$; T: pret.; M: ind.; A: imp.; Tr: acc.; N: sing.

NE.: plundered, was...devastating

$122 / 10$ beon forsugiende (...) secgende

C: infinitive; A: perf.; Tr: gen.

NE.: pass over, be silent

C: infinitive; A: imp.; Tr: gen.

NE.: dwell upon, be speaking

124/9 waes farende

C: $\mathrm{m}+$; T: pret.; M: ind.; A: imp.; Tr: intran.; N: sing. NE.: marched, marched

124/35 waes ondraedende

C: $\mathrm{m}+$; T: pret.; M: ind.; A: imp.; Tr: prep.; N: sing.

NE.: dreaded, dreaded

128/8 waes fleonde

C: sub. +; T: pret.; M: ind.; A: imp.; Tr: intra.; N: sing.

NE.: fled, fled

130/9 winnende waes

C: sub. +; T: pret.; M: ind.; A: imp.; Tr: intran.; N: sing.

NE.: continued, was...carrying on war

$130 / 20$ waeron farende

C: sub. +; T: pret.; M: ind.; A: imp.; Tr: intran.; N: pl. NE.: marched with him, his associated

$130 / 21$ waeron winnende

C: sub. +; T: pret.; M: ind.; A: imp.; Tr: intran.; N: pl. NE.: marched, fellow-warriors

130/30 hienende waes

C: sub. +; T: pret.; M: ind.; A: imp.; Tr: acc.; N: sing.

NE.: was...killing, was the oppressor 
130/31 waes sinpyrstende

C: $\mathrm{m}+$; T: pret.; M: ind.; A: imp.; Tr: gen.; N: sing.

NE.: was thirsty, was...thirsting

$132 / 24$ waes feohtende

C: sub.+; T: pret.; M: ind.; A: imp.; Tr: prep.; N: sing.

NE.: was opposing, had fought

134/7 waeron dreogende

C: $\mathrm{m}+$; T: pret.; M: ind.; A: imp.; Tr: acc.; N: pl.

NE.: it was a long time, were long contending

134/14 waeran ehtende

C: $\mathrm{m}+$; T: pret.; M: ind.; A: imp.; Tr: gen.; N: pl.

NE.: attacked, assailed

140/27 dreogende waeron

C: sub. +; T: pret.; M: ind.; A: imp.; Tr: acc.; N: pl.

NE.: had continued, they had been carrying

148/2 waes donde

C: $\mathrm{m}+$; T: pret.; M: ind.; A: imp.; Tr: acc.; N: sing.

NE.: distressed, did...evil

$148 / 35$ winnende waeron

C: $\mathrm{m}+$; T: pret.; M: ind.; A: imp.; Tr: prep.; N: pl.

NE.: carry on the war, made war

154/34 begon(gen)de waes

C: sub. +; T: pret.; M: ind.; A: imp.; Tr: acc.; N: sing.

NE.: -, worshipped

158/10 waeron sleande

C: $\mathrm{m}+$; T: pret.; M: ind.; A: imp.; Tr: acc.; N: pl.

NE.: slew, slew

$158 / 27$ waeron sleande

C: sub. +; T: pret.; M: ind.; A: imp.; Tr: acc.; N: pl.

NE.: would turn against, slew

$160 / 6$ winnende waes

C: sub.+; T: pret.; M: Ind.; A: imp.; Tr: prep.; N: sing.

NE.: war continued, was warring 


\section{$162 / 27$ laerende waeron}

C: sub.+; T: pret.; M: ind.; A: imp.; Tr: dat./acc.; N: pl.

NE.: taught, taught

166/12 waeron acsiende 7 frinende

C: $\mathrm{m}+$; T: pret.; M: ind.; A: imp.; Tr: prep.; N: pl.

NE.: asked, (was) asking

C: $\mathrm{m}+$; T: pret.; M: ind.; A: imp.; Tr: prep.; N: pl.

NE.: asked, (was) inquiring

$166 / 19$ wैaes braedende

C: $\mathrm{m}+$; T: pret.; $\mathrm{M}$ : ind.; A: imp.; Tr: prep.; $\mathrm{N}$ : sing.

NE.: raised, streched

$166 / 20$ waes waniende

C: $\mathrm{m}+$; T: pret.; M: ind.; A: imp.; Tr: acc.; N: sing.

NE.: bemoaning, bewailed

$166 / 25$ waes girnende

C: ambiguous + ; T: pret.; M: ind.; A: imp.; Tr: gen.; N: sing.

NE.: was ambitious, was craving after

168/17 fremmende waere

C: sub. +; T: pret.; M: subj.; A: imp.; Tr: gen.; N: sing.

NE.: had plotted, of having acted

168/29 waes sleande 7 hienende

C: $\mathrm{m}+$; T: pret.; $\mathrm{M}$ : ind.; A: imp.; Tr: acc.; N: sing.

NE.: slew, was driving out

C: $\mathrm{m}+$; T: pret.; M: ind.; A: imp.; Tr: acc.; N: sing.

NE.: made depredation, (was) oppressing

168/32 aefterfylgende waes

C: $\mathrm{m}$ + ; T: pret.; M: ind.; A: imp.; Tr: dat.; N: sing.

NE.: pursued, followed after

168/34 waes hergende 7 baernende

C: $\mathrm{m}+$; T: pret.; M: ind.; A: imp.; Tr: intran.; N: sing.

NE.: harassed, was harrying

C: $\mathrm{m}+$; T: pret.; M: ind.; A: imp.; Tr: intran.; N: sing.

NE.: burned, (was) burning 
m 1 ) T: pret.; M: ind.; A: imp.; Tr: intran.; N: sig.

NE. Was advancing, was marching

$172 / 22$ waeron feohtende

C: sub. +; T: pret.; M: ind.; A: imp.; Tr: prep.; N: pl.

NE.: - were...fighting

$172 / 34$ waeron hergende

C: $\mathrm{m}+$; T: pret.; M: ind.; A: imp.; Tr: intran.; N: pl.

NE.: laid waste, harried

$174 / 2$ farende waes

C: sub. +; T: pret.; M: ind.; A: imp.; Tr: intran.; N: sing.

NE.: - , he came

$182 / 4$ dreogende waeron

C: $\mathrm{m}+$; T: pret.; M: ind.; A: imp.; Tr: acc.; N: pl.

NE.: occasioned, endured

184/3 onwinnende waes

C: $\mathrm{m}+$; T: pret.; M: ind.; A: imp.; Tr: dat.; N: sing.

NE.: being now attacked, was raging

186/4 waes sittende

C: $\mathrm{m}+$; $\mathrm{T}$ : pret.; $\mathrm{M}$ : ind.; A: imp.; Tr: intran.; N: sing.

NE.: continued, was sitting

186/11 waes wenende

C: sub. +; T: pret.; M: ind.; A: imp.; Tr: obj.c.; N: sing.

NE.: conceived, imagined

188/12 farende waes

C: $\mathrm{m}+$; T: pret.; M: ind.; A: imp.; Tr: intran.; N: sing.

NE.: approaching, proceeded

183/13 waes bencende

C: $\mathrm{m}+$; T: pret.; $\mathrm{M}$ : ind.; A: imp.; Tr: obj.c.; $\mathrm{N}$ : sing.

NE.: with intention, thought

$188 / 19$ waes donde

C: $\mathrm{m}+$; T: pret.; M: ind.; A: imp.; Tr: acc.; N: sing.

NE.: had many engagements, fought many battles 
190/4 waeron aefterfylgende 7 (...) sleande

C: $\mathrm{m}+$; T: pret.; M: ind.; A: imp.; Tr: dat.; N: pl.

NE.: pursued, pursued

C: $\mathrm{m}+$; T: pret.; M: ind.; A: imp.; Tr: ac.; N: pl.

NE.: killing, slew

194/22 wilniende waes 7 wenende

C: sub. +; T: pret.; M: ind.; A: imp.; Tr: gen.; N: sing.

NE.: had hoped, was desirous

C: sub. +; T: pret.; M: ind.; A: imp.; Tr: gen.; N: sing.

NE.: had hoped, was...hoping

196/13 waes biddende

C: $\mathrm{m}+$; T: pret.; $\mathrm{M}$ : ind.; A: imp.; Tr: obj.c.; N: sing.

NE.: pressed, entreated

200/20 waes sleande

C: $\mathrm{m}+$; T: pret.; M: ind.; A: imp.; Tr: acc.; N: sing.

NE.: made a carnage, was...slaying

202/10 biddende waes

C: $\mathrm{m}+$; T: pret.; M: ind.; A: imp.; Tr: obj.c.; N: sing.

NE.: desired, prayed

202/10 wilniende waes

C: $\mathrm{m}+$; T: pret.; M: ind.; A: imp.; Tr: obj.c.; N. sing.

NE: desired, desired

202/31 dreogende waeron

C: sub.+; T: pret.; M: ind.; A: imp.; Tr: acc.; N. pl.

NE.: had continued, had been carrying

208/13 waes swencende

C: $\mathrm{m}+$; T: pret.; $\mathrm{M}$ : ind.; A: imp.; Tr: acc.; $\mathrm{N}$ : sing.

NE.: swinged, harassed

212/4 waes feohtende

C: $\mathrm{m}+$; T: pret.; M: ind.; A: imp.; Tr: prep.; N: sing.

NE.: continued his attacks, was fighting

\section{4/15 waeron dreogende}

C: sub. +; T: pret.; M: ind.; A: imp.; Tr: acc.; N: pl.

NE.: suffered, were suffering 
$216 / 15$ weard aefterfylgende

C: $\mathrm{m}$ +; T: pret.; M: ind.; A: imp.; Tr: dat.; N: sing.

NE.: - , -

218/30 waes winnende

C: $\mathrm{m}$ +; T: pret.; M: Ind.; A: imp.; Tr: intran.; N: sing.

NE.: having carried on the war, was there warring

$220 / 2$ waes wuniende

C: $\mathrm{m}+$; $\mathrm{T}$ : pret.; M: ind.; A: imp.; Tr: intran.; N: sing.

NE.: continued, continued

222/6 irnende waeron

C: $\mathrm{m}+$; T: pret.; M: ind.; A: imp.; Tr: intran.; N: pl.

NE.: issued forth, ran out

224/28 dreogende waes

C: $\mathrm{m}+$; T: pret.; M: ind.; A: imp.; Tr: acc.; N: sing.

NE.: he had undergone, he had undergone

226/6 faerende waeron

C: sub + ; T: pret.; M: ind.; A: imp.; Tr: intran.; N: pl.

NE.: the ships, were sailing

230/17 waeron pafiende

C: $\mathrm{m}+$; T: pret.; M: ind.; A: imp.; Tr: acc.; N: pl.

NE.: obliged to endure, were enduring

230/18 waeron donde

C: $\mathrm{m}+$; $\mathrm{T}$ : pret.; M: ind.; A: imp.; Tr: acc.; N: pl.

NE.: continued, they were doig

232/21 waes oppyncende

C: $\mathrm{m}+$; T: pret.; M: ind.; A: imp.; Tr: dat.; N: sing.

NE.: was not approved, taking this ...ill

232/24/11 waeron biddende

C: $\mathrm{m}+$; T: pret.; M: ind.; A: imp.; Tr: obj.c.; N: pl.

NE.: proposed, requested

232/27 waes weaxende

$\mathrm{C}: \mathrm{m}+$; T: pret.; M: ind.; A: imp.; Tr: intran.; N: sing.

NE.: grew, was increased 
234/8 waes biernende

C: $\mathrm{m}+$; $\mathrm{T}$ : pret.; $\mathrm{M}$ : ind.; A: imp.; Tr: intran.; N: sing.

NE.: ascended, burnt up

234/10 waes bradiende

C: $\mathrm{m}+$; T: pret.; M: ind.; A: imp.; Tr: intran.; N. sing.

NE.: declined, extending

234/11 waes farende

C: $\mathrm{m}+$; $\mathrm{T}$ : pret.; M: ind.; A: imp.; Tr: intran.; N: sing.

NE.: rose, returned

236/9 farende waes

C: $\mathrm{m}+$; T: pret.; M: ind.; A: imp.; Tr: intran.; N. sing.

NE.: marched, marched

236/15 waes cirrende

C: $\mathrm{m}+$; T: pret.; M: ind.; A: imp.; Tr: intran.; $\mathrm{N}$ : sing.

NE.: proceeded, quickly again turned

236/20 waes farende

C: $\mathrm{m}+$; T: pret.; M: ind.; A: imp.; Tr: intran.; N: sing.

NE.: returned, proceeded

236/29 aefterfylgende waes

C: $\mathrm{m}+$; T: pret.; M: ind.; A: imp.; Tr: dat.; N: sing.

NE.: pursued, pursued

238/3 waeron dreogende

C: sub. +; T: pret.; M: ind.; A: imp.; Tr: acc.; N: pl.

NE.: was suffered, they endured

242/19 waes maenende

C: $\mathrm{m}+$; $\mathrm{T}$ : pret.; $\mathrm{M}$ : ind.; A: imp.; Tr: acc.; N: sing.

NE.: wept, bewailed

244/4 waes waniende

C: $\mathrm{m}+$; $\mathrm{T}$ : pret.; $\mathrm{M}$ : ind.; A: imp.; Tr: obj.c.; N: sing.

NE.: conceived, grieved

246/7 waeron farende

C: ambiguous +; T: pret.; M: ind.; A: imp.; Tr: intran.; N: pl.

NE.: were embarked, were faring 
246/8 waeron farende

C: ambiguous +; T: pret.; M: ind.; A: imp.; Tr: intran.; N: pl.

NE.: he had, were faring

\section{2/12 waes micliende}

C: $\mathrm{m}+$; T: pret.; $\mathrm{M}$ : ind.; A: imp.; Tr: intran.; N: sing.

NE.: continued, was...increasing

$260 / 29$ donde waes

C: sub. +; T: pret.; M: ind.; A: imp.; Tr: acc.; N: sing.

NE.: - in addition ...he perpetrated

262/2 waes biernende

C: $\mathrm{m}+$; $\mathrm{T}$ : pret.; $\mathrm{M}$ : ind.; A: imp.; Tr: intran.; $\mathrm{N}$ : sing.

NE.: continued burning, that was burning

274/19 beon winnende

C: infinitive; A: imp.; Tr: intran.

NE.: - , be ever warring

274/27 waeron onwinnende

C: $\mathrm{m}+$; T: pret.; M: ind.; A: imp.; Tr: dat.; N: pl.

NE.: made war, made war

278/24, waeron winnende

C: $\mathrm{m}+$; T: pret.; M: ind.; A: imp.; Tr: prep./dat.; N: pl.

NE.: declared war, were...making war

280/29 waes giernende

C: $\mathrm{m}+$; T: pret.; M: ind.; A: imp.; Tr: gen.; N: sing.

NE.: was...covetous, little desirous

284/31 waes farende

C: $\mathrm{m}+$; T: pret.; $\mathrm{M}$ : ind.; A: imp.; Tr: intran.; N: sing.

NE.: marched, went

290/7 waes wilniende

C: ambiguous + ; T: pret.; M: ind.; A: imp.; Tr: gen.; N: sing.

NE.: - , -

292/23 waes bencende

C: $\mathrm{m}+$; pret.; $\mathrm{M}$ : ind.; A: imp.; Tr: obj.c.; $\mathrm{N}$ : sing.

NE.: was...desirous, was thinking 
296/13 waes blotende

C: $\mathrm{m}+$; T: pret.; $\mathrm{M}$ : ind.; A: imp.; Tr.: dat.; N: sing.

NE.: offered, sacrified

\subsubsection{Statistical results}

\subsubsection{Expanded tenses (ET)}

total: 237

finite forms: $231^{9}$

infinitives: $6(2.5 \%)$

in affirmative clauses: 229

in non-affirmative clauses: 2

$P(a 1)=0.8 \%$

in main clauses: 152

in subordinate clauses: 70

$P(a 2)=30.3 \%$

ambiguous: 9

In present tense: 16

in preterite: 215

$$
P(a 3)=93.1 \%
$$

in indicative: 219

in subjunctive: 12

$$
P(a 4)=5.2 \%
$$

in perfective aspect: 3

in imperfective aspect: 234

$P(a 5)=98.7 \%$

with accusative: 62

intransitive: 83

with prepositional object: 39

with object clause: 20

with genetive: 16

with dative: 20

total marked transitivity: 95

$P(a 6)=40.1 \%$

in singular: 127

in plural: 104

$P(a 7)=45.0 \%$

9 Bold print indicates favourable events. 


\subsubsection{Non-expanded tenses (NET)}

total analysed clauses: 855

finite forms: 800

infinitives: $55(6.4 \%)$

in affirmative clauses: $\mathbf{7 5 3}$

in non-affirmative clauses: 40

$$
P(b 1)=5.0 \%
$$

in main clauses: 483

in subordinate clauses: 317

$$
P(b 2)=39.6 \%
$$

in present tense: 124

in preterite: 676

$$
P(b 3)=84.5 \%
$$

in indicative: 732

in subjunctive: 68

$$
P(b 4)=8.4 \%
$$

in perfective aspect: 442

in imperfective aspect: 358

$$
P(b 5)=45.0 \%
$$

with accusative: $326(38.1 \%)$

intransitive: 253

with prepositional object: 52

with object clause: 69

with genetive: 68

with dative: 87

total marked transitivity: 276

$P(b 6)=32.3 \%$

in singular: 516

in plural: 284

$$
P(b 7)=35.5 \%
$$

\subsubsection{The comparison of the basic sample and the control sample}

The probability rates of favourable events (the presence of marked parameters) are higher in the basic sample for the parameters tense $(93.1 \%: 84.5 \%)$, aspect $(98.7 \%: 45.0 \%)$, transitivity $(40.1 \%: 32.3 \%)$ and number $(45.0 \%: 35.5 \%)$. The probability rates of favourable events are lower in the basic sample for the parameters propositional modality $(0.8 \%: 5.0 \%)$, type of clause $(30.3 \%: 39.6 \%)$ and $\operatorname{mood}(5.2 \%: 8.5 \%)$.

The probability of a (non)expanded tense to assume a finite form (favourable event) is higher in the basic sample than in the control sample $(97.5 \%: 93.6 \%)$. 
The following table shows the probability rates of favourable events (the marked parameter value) in both samples. The index "Id" shows whether the difference between two probability rates (e.g. $\mathrm{Pa} 1$ and $\mathrm{Pb} 1$ ) is statistically significant.

marked values of parameters $\begin{gathered}\text { probability rates } \\ \text { in basic sample }\end{gathered} \quad \begin{gathered}\text { probability rates } \\ \text { in control sample }\end{gathered} \quad$ Index Id

\begin{tabular}{lllr}
\hline 1. non-affirmative clause: & P(a1): $0.8 \%$ & P(b1): $5.0 \%$ & 2.8 \\
2. subordinate clause: & P(a2): $30.3 \%$ & P(b2): $39.6 \%$ & 2.6 \\
3. preterite: & P(a3): $93.1 \%$ & P(b3): $84.5 \%$ & 3.3 \\
4. subjunctive: & P(a4): 5.2\% & P(b4): $8.5 \%$ & 1.7 \\
5. imperfective: & P(a5): $98.7 \%$ & P(b5): $45.0 \%$ & 14.7 \\
6. marked transitivity: & P(a6): $40.1 \%$ & P(b6): $32.3 \%$ & 2.3 \\
7. plural: & P(a7): $45.0 \%$ & P(b7): $35.5 \%$ & 2.7
\end{tabular}

"Cumulative markedness"

The parameters studied assume a marked value

ET NET

\begin{tabular}{lll}
\hline in at least $25 \%$ of the clauses & 5 parameters & 5 parameters \\
in at least $50 \%$ of the clauses & 2 parameters & 1 parameters \\
in at least $75 \%$ of the clauses & 2 parameters & 1 parameters
\end{tabular}

or, the marked value can be expected for

\begin{tabular}{|c|c|c|}
\hline one grammatical category & $98.7 \%$ of ET & $84.7 \%$ of NET \\
\hline two grammatical categories & $93.1 \%$ & $45.0 \%$ \\
\hline three grammatical categories & $59.9 \%$ & $39.6 \%$ \\
\hline four grammatical categories & $45.0 \%$ & $35.5 \%$ \\
\hline five grammatical categories & $40.1 \%$ & $32.3 \%$ \\
\hline six grammatical categories & $5.2 \%$ & $8.5 \%$ \\
\hline seven grammatical categories & $0.8 \%$ & $5.0 \%$ \\
\hline
\end{tabular}

The mean probability rate of the marked values is

$\mathrm{P}(\mathrm{ET})=44.7 \% \quad \mathrm{P}(\mathrm{NET})=35.8 \%$

\subsection{THE FURTHER DEVELOPMENT OF ENGLISH EXPANDED TENSES}

2.4.1. Expanded tenses were rather infrequent in Old English. While a number of scholars tried to discover the rules of the usage of expanded tenses in Old English, recent studies claim that expanded tenses were used promiscue with the non-expanded tenses and that the actual usage of one or the other syntactic variant depended on the environment of the verbal phrase. Their emphatic value seems rather obvious, though, which explains to some extent that expanded tenses were more frequent in vivid historic descriptions than in philosophical prose (Nickel 1966, Kisbye 1971). 
2.4.2. In Middle English the use of expanded tenses declined, except in the northern dialects. From there it spread again towards the South after the year 1200, where it gained grounds especially after 1400 . The reassertion of expanded tenses has been ascribed to various factors. Some authors stress the importance of the influence of French and of the Celtic languages (Mustanoja 1960), as well as the analogy with the construction "be + a- +- ing" (from the older construction "be + on +- -ing"). The incidence of the "be $+\mathrm{a}-+-$-ing" construction was vastly inferior to the one with "be + -ing", even when the former was in its peak period $(1500-1700)$ (Kisbye 1971, 36-38). About the year 1200, the ending -ing started to compete with ende in the southern dialects. From there it spread to other parts of England and ultimately prevailed sometime after the year 1500 . The unification of endings must have been enhanced by the phonetic instability of a multitude of endings (-en, -enne, -ing, -ung), and even more so by the stable ending -ing (-ung) in the gerund.

The use of expanded tenses in Middle English did not differ very much from their use in Old English. The use was still not regularized, although some characteristics seem to stand out: the durative, emphatic and emotional force of expanded tenses if compared with the corresponding non-expanded tenses. In the late Middle English period the expanded form of the verb "come" began to denote future time. The same period gave rise to the construction "be + going to + verb".

2.4.3. In the 16 th century, the use of expanded tenses grew more stable and has been spreading ever since, although expanded tenses still account for only $5 \%$ of verbal phrases in Modern English (Quirk et al. 1985). In the early 18th century the use of expanded tenses was regularized at practically the present state of affairs.

In Modern English, the use of expanded tenses is

a) obligatory

- to express the imperfective aspect as opposed to non-expanded tenses, which do not express this aspect. Here the imperfective aspect relates to the progressive mode of action; it describes the verbal situation in its progress, adding to the semantic value of the verb the information "+ progressive" and "+durative";

- to express the temporary value of the verbal situation, adding to the semantic information of the verb the feature "+ temporary";

- to encode the simultaneity of the verbal situation with another situation, hence the additional "+ simultaneous" information.

It is often difficult to tell the above functions of expanded tenses apart. 
b) optional

- to express a repetative situation (with adverbs like always, continually, constatly...), in which case the expanded tenses contribute " + emotional colouring";

"she is always quarrelling..." (+ annoyance)

"he will always be dreaming..." (+ condescendence)

c) exceptional

- with static verbs denoting perception, mental activity, emotions and situations. Such verbs are used to describe situations which are beyond our will or control, they refer to state rather than to action. When applied, the expanded tenses turn the verbs from static to dynamic, or else emphasize some other specific attribute (e.g. gradualness, emphasis):

"I am feeling the blanket and it feels soft"

"Do you like it? No, I'm hating it"

"I study mathematics and every day I am learning more".

d) impossible

- with some static verbs the use of expanded tenses is not possible, not even in exceptional cases (e.g. know); the same applies to the use of expanded tenses with modal verbs.
" I am knowing you more and more"
“ you are maying go“.

\section{CONCLUDING REMARKS}

\subsection{THE COMPARISON OF THE WORKING HYPOTHESIS WITH THE RESULTS OF THE ANALYSIS OF OROSIUS}

\subsubsection{Prediction:}

Expanded tenses asserted themselves in Old English first in a relatively complex linguistic environment

a) The prediction that strengthenings assert themselves first with complex ("heavy") words cannot be tested in our material and can therefore be neither con- 
firmed nor rejected. The most frequent verbs in our basic sample are (Nickel 1966, 330-351):

winnan (to make war)

faran (to travel)

dreogan (to continue)

aefterfylgan (to pursue)

don (to do)

feohtan (to fight)

hienan (to oppress)

irnan (to run)
34 (of 237 instances)

16

13

10

10

10

10

10.

These verbs are the most frequent ones in the control sample, too, owing to the contents of the text (history and geography). The judgement of their "complexity" could not be objective.

b) The results of the statistical analysis confirm the prediction that expanded tenses are more frequent in a complex environment. Of the seven parameters observed (person and voice are left out due to the absence of non-third persons and passive voice), four parameters (tense, aspect, transitivity and number) assume their marked value significantly more often in the sample of expanded tenses than in the sample of non-expanded tenses.

The fact that the working hypothesis is not confirmed in the case of propositional modality and mood does not necessarily bear much weight, considering the low frequency of negative clauses and of subjunctive mood, both in the basic and in the control sample (bellow 10\%).

Even more indicative than the comparison of the basic and of the control samples in terms of individual parameters is the comparison of both samples as to the "cumulative markedness" of the clauses/verbal phrases in which (non)-expanded tenses occur. Our working hypothesis is sustained in the sense that in more than $50 \%$ of the instances of the basic sample three grammatical parameters assume their marked value, while only one grammatical parameter assumes its marked value in more than $50 \%$ of the instances in the control sample. The mean probability rate of a grammatical parameter to assume a marked value (arithmetic mean) is higher in the sample of expanded tenses than in the sample of non-expanded tenses, which also speaks in favour of our working hypothesis.

\subsubsection{Prediction:}

The grammaticalization of the use of expanded tenses was to result in their specialization for the encoding of more marked (complex) information. The areas to 
which the use of expanded tenses has not spread are simple (unmarked) contexts and situations.

The prediction does obtain in the light of the following:

a) In Modern English, the use of expanded tenses is obligatory to express the marked verbal aspect and mode of verbal action, i.e. progressiveness, temporary duration, simultaneity, ingressiveness, imperfectiveness, etc.

b) Whenever the use of expanded tenses is optional, meaning that they still act as the strengthenings of the respective non-expanded tenses, the expanded tenses imply emotional colouring, or stress a particular mode of verbal action.

c) Very frequent and relatively simple verbs, such as static verbs (of perception, emotions, mental activity), are used in expanded tenses only exceptionally. Some verbs change their lexical meaning when assuming the expanded form, so that they turn from static verbs into verbs denoting actions, the action being considered more marked than the state.

d) The use of expanded tenses has not spread to some of the most frequent verbs, such as modal verbs.

\subsection{GENERAL ASSESSMENT OF THE WORKING HYPOTHESIS AND POSSIBILITIES FOR FURTHER RESEARCH}

The analysis of the Old English Orosius and the survey of the use of expanded tenses in Modern English indicate that the consideration of syntactic changes as strengthenings or weakenings deserves serious attention. The results of our statistical analysis need to be compared with the results of the analyses not only of additional texts, but also of other syntactic changes, possibly in other languages as well. If such comparisons confirm the theory of weekenings and strengthenings in syntax (the theory would then fit the notion of "natural syntax" - by analogy with "natural phonology" and "natural morphology"), it would be most interesting to find out whether and how the behaviour of syntactic strengthenings/weakenings differs from the behaviour of phological or morphological strengthenings/weakenings.

Syntax is likely to tell us a great deal about the functioning of the human mind; the principles governing syntactic changes viewed as a response to communication problems can be one of the keys to the communication between man and artificial intelligence. 
Bosworth, J., ed. King Alfred's Anglo-Saxon version of the compendious history of the World. London, 1859.

Klaeber, F., ed., Beowulf and The fight at Finnsburg. Boston, 1950, 3rd ed.

Mayerthaler, W., Morphologische Natuerlichkeit. Wiesbaden, 1981.

Mossé, F., Histoire de la forme périphrastique être + participe présent en germanique, 1-2. Paris, 1938.

Mustanoja, T.F., A Middle English syntax I. Helsinki, 1960.

Nickel, G., Die Expanded Form im Altenglischen. Vorkommen, Funktion und Herkunft beon/wesan + Partizip praesens. Neumuenster, 1966.

Pavlić, I., Statistička teorija i primjena. Zagreb, 1985.

Sweet, H., ed., King Alfred's Orosius, EETS Nr. 79. London, 1883, reprinted 1959. Wise, F., ed., Annales rerum gestarum Aelfredi Magni, auctore Asserio Menevensi. Oxford, 1722.

\section{Povzetek \\ RAZŠIRJENI ČASI V STAROANGLESKKEM OROSIJU: SKLADENJSKA OKREPITEV}

Razširjeni časi (opisne zgradbe, ki so sestavljene iz pomožnega glagola 'biti' in deležnika sedanjika) so se v stari angleščni uporabljali kot skladenjska različica ustreznih nerazširjenih časov (nesestavljenih glagolskih zgradb). Njihova raba je bila poljubna, bili so nosilci enakih slovničnih pomenov kot ustrezni nerazširjeni časi.

Vprašanje, na katerega je želela odgovoriti avtorica, je, ali se je slovnično okolje staroangleških razširjenih časov statistično signifikantno razlikovalo od okolja ustreznih staroangleških nerazširjenih časov. S tem namenom je bila opravljena primerjalna analiza stavkov in skladnje glagolov, ki se pojavljajo v razširjenih oz. nerazširjenih časih v staroangleškem prevodu knjige španskega redovnika Orosija o zgodovini sveta.

Analiza je zajela opazovanje sedmih slovničnih kategorij (oblika in vrsta stavka, glagolski čas, naklon, vid, prehodnost, število) s stališca njihove bolj ali manj zapletene (označene) vrednosti, ki jo imajo $v$ primeru rabe razširjenih časov (osnovni vzorec) oziroma nerazširjenih časov (kontrolni vzorec).

V obdelanem jezikovnem gradivu so se razširjeni časi pogosteje pojavljali v pretekliku kot $v$ sedanjiku, v nedovršnem glagolskem vidu kot $v$ dovršnem, $v$ množini kot $v$ ednini, $v$ zapleteni prehodnosti pogosteje kot z golim tožilnikom. Stopnja verjetnosti, da se več slovničnih kategorij pojavi istočasno v zapleteni vrednosti, je večja pri razširjenih časih kot pri nerazširjenih časih. Aritmetična sredina pogostnosti označenih vrednosti slovničnih kategorij je višja v osnovnem vzorcu (razširjeni časi) kot v kontrolnem vzorcu (nerazširjeni časi).

Primerjava rezultatov statistične obdelave jezikovnega gradiva $z$ delovnimi napovedmi je pokazala, da so staroangleški razširjeni časi v obdelanem jezikovnem gradivu vedli v primerjavi $z$ nerazširjenimi časi kot okrepitev ter da je torej tudi skladenjske spremembe smotrno obravnavati s stališca teorije o jezikovnih spremembah kot okrepitvah ali ošibitvah. 